\title{
Transient Pseudohypoaldosteronism in an Infant: A Case Report*
}

\author{
Tin Nwe Latt, ${ }^{1}$ Siti Iryawani Rahman, ${ }^{2}$ Noor Shafina Mohd Nor ${ }^{1}$ \\ ${ }^{1}$ Faculty of Medicine, Universiti Teknologi MARA, Sungai Buloh, Selangor, Malaysia \\ ${ }^{2}$ Hospital Sungai Buloh, Jalan Hospital, Sungai Buloh, Selangor, Malaysia
}

\begin{abstract}
Transient pseudohypoaldosteronism is strongly linked to urinary tract infections complicating structural urinary tract anomalies. A 3-month-old baby girl with hyponatremia, hyperkalemia and metabolic acidosis associated with urinary tract infection and structural urinary tract anomalies was diagnosed with transient pseudohypoaldosteronism following elevated serum aldosterone and normal 17-hydroxyprogesterone level. Electrolytes normalized with corrections and antibiotic therapy. Clinicians should have a high index of suspicion for transient pseudohypoaldosteronism in an infant presenting with hyponatremia, hyperkalemia and urinary tract infection with or without associated urinary tract anomalies.
\end{abstract}

Key words: pseudohypoaldosteronism, urinary tract infection (UTI), urinary tract anomalies

\section{INTRODUCTION}

Electrolyte imbalances, such as hyponatremia and hyperkalemia, are emergencies, especially in small infants. These abnormalities may be due to impaired mineralocorticoid secretion or response. Some causes to be considered are congenital adrenal hyperplasia (CAH) or hypoplasia, isolated aldosterone deficiency, certain medications or pseudohypoaldosteronism (PHA), a rare syndrome of mineralocorticoid resistance. ${ }^{1}$

PHA can be classified into Type 1 and Type 2. Type 1 PHA is a rare condition characterized by renal resistance to the actions of aldosterone and is further sub-classified into primary and secondary (transient) PHA. $^{2}$ In Type 1 primary PHA, there is mutation in the mineralocorticoid receptor that causes end organ resistance to mineralocorticoids. Type 1 secondary or transient PHA is strongly associated with urinary tract infections (UTI) complicating structural urinary tract anomalies. ${ }^{2,3}$

We report a case of transient PHA in a 3-month-old baby with hyponatremic hyperkalemic metabolic acidosis to highlight that although rare, transient PHA should be considered as a differential diagnosis of hyponatremia and hyperkalemia in infants with UTI complicating structural urinary tract anomalies. Furthermore, it also points out that the existence of urinary tract infection complicating urinary tract malformations is a powerful predisposition for the development of transient aldosterone resistance.

\section{CASE}

A 3-month-old, previously well Chinese girl, presented with one-week history of diarrhoea followed by one episode of afebrile seizure. She was referred from a district hospital for further work up and management. Her birth history was also unremarkable. She was delivered at term with birth weight of $3.72 \mathrm{~kg}$ ( $75^{\text {th }}$ centile) and length of $55 \mathrm{~cm}$ (97 th centile) (Figure 1). She had been on exclusive breastfeeding for 1 month and then changed to infant formula. She was on multiple different brands of cow's milk formula in view of recurrent episodes of loose stool and excessive flatulence.

On examination, she was moderately dehydrated and tachycardic. Her weight was $6.2 \mathrm{~kg}$ (above $75^{\text {th }}$ centile) and length of $61 \mathrm{~cm}$ ( $75^{\text {th }}$ centile). Examination revealed ballotable right kidney. Initial laboratory investigations showed hyponatremia (117 $\mathrm{mmol} / \mathrm{L})$, hypochloremia $(90$ mmol/l), hyperkalemia (9 $\mathrm{mmol} / \mathrm{L})$ with metabolic acidosis. She required intravenous (IV) normal saline bolus $(10 \mathrm{ml} / \mathrm{kg})$ given at the district hospital and IV hydration with $5 \%$ correction over 12 hours. She was given one cycle of lytic cocktail with calcium resonium 6 hourly to correct the hyperkalemia. Her electrolytes normalized after 4 days of treatment. Stool culture for rotavirus and reducing sugar were negative. In view of the possibility of cow's milk protein allergy, she was commenced on extensive hydrolysed milk formula and subsequently responded well with resolution of
ISSN 0857-1074 (Print) I ISSN 2308-118x (Online) Printed in the Philippines

Copyright (C) 2018 by the JAFES

Received: January 16, 2018. Accepted: March 12, 2018.

Published online first: April 12, 2018.

https://doi.org/10.15605/jafes.033.01.07

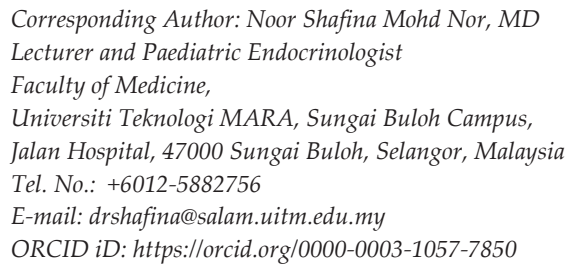


diarrhoea. She had no recurrent episode of seizure and her neurological examination was unremarkable with normal ultrasound scan of the brain.

Her urine culture grew Enterobacter and therefore, she was treated with 7 days course of cefuroxime. Ultrasound of kidney, ureter, and bladder (USG KUB) showed right duplex kidney with gross hydronephrosis and hydroureter, while a micturating cystourethrogram (MCUG) showed grade 5 vesicoureteric reflux on the right side.

Birth to 36 months: Girls

Length-for-age and Weight-for-age percentiles

NAME RECORD \#

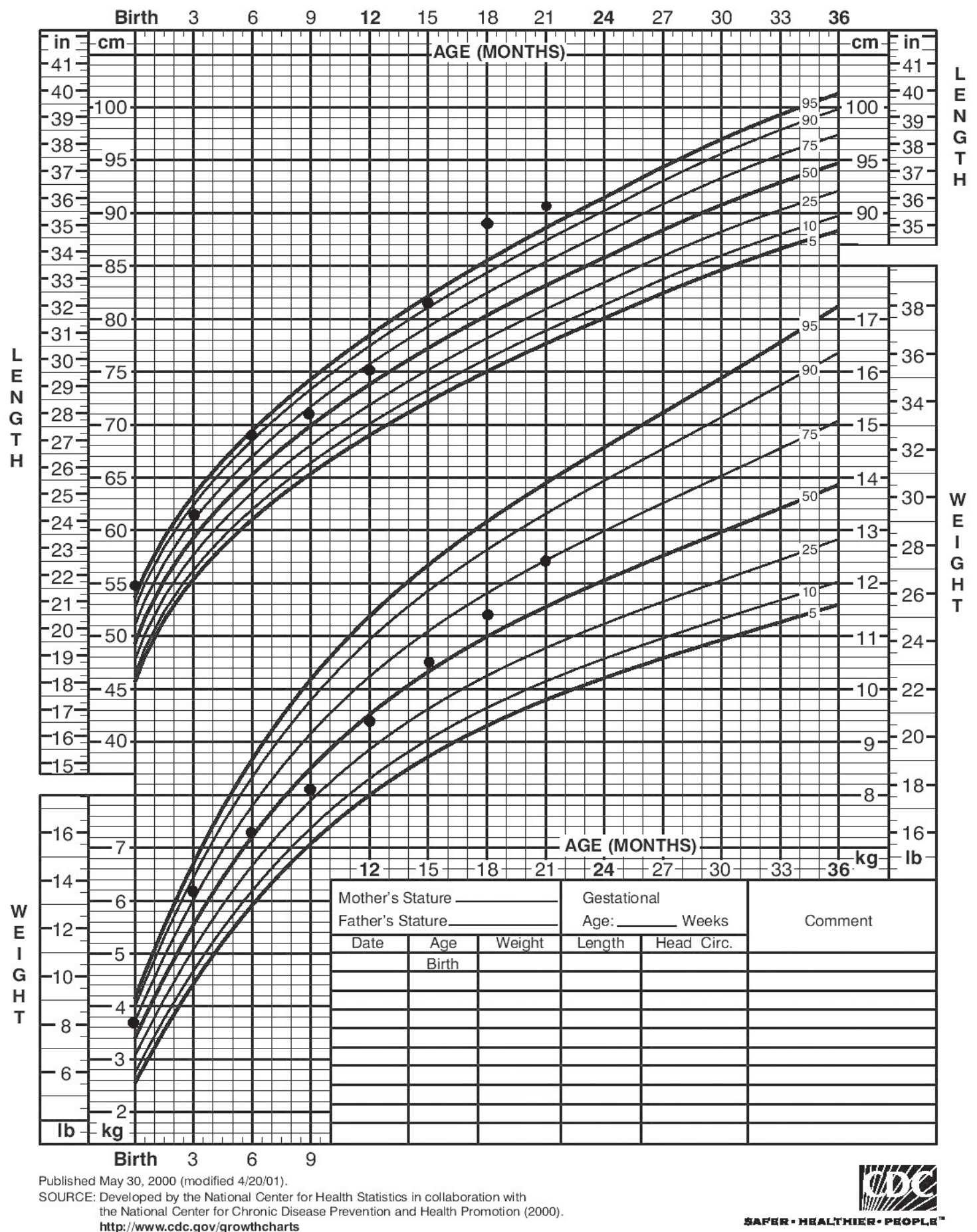

Figure 1. Growth chart from birth to 2 years old (downloaded from https://www.cdc.gov/growthcharts/clinical_charts.htm). 
Routine screening for $\mathrm{CAH}$ is not done in our country. The diagnosis of transient PHA became apparent when the serum aldosterone level obtained on admission was elevated at $3700 \mathrm{pmol} / \mathrm{l}$ (normal range $<1109 \mathrm{pmol} / \mathrm{L}$ ) with normal 17-hydroxyprogesterone (17-OHP) level (0.8 $\mathrm{nmol} / \mathrm{L}$, reference range 1.0-14.12 $\mathrm{nmol} / \mathrm{L})$. Adrenocorticotropic hormone $(\mathrm{ACTH})$ stimulation test was also normal. She was commenced on oral trimethoprim for urinary tract prophylaxis prior to discharge. She has no subsequent recurrent episode of electrolyte disturbances nor urinary tract infection as evidenced by negative urine culture post treatment. She is now two years old and currently under our follow up. Her growth is satisfactory with weight $12.4 \mathrm{~kg}$ (above $50^{\text {th }}$ centile) and height $90 \mathrm{~cm} \quad\left(90^{\text {th }}\right.$ centile). Her developmental milestones are appropriate and she is currently awaiting urethral implantation.

\section{DISCUSSION}

The primary function of aldosterone is reabsorption of sodium and water at the expense of potassium in the distal renal tubule. Deficiency of aldosterone or end organ resistance to its actions leads to hyponatremia, hyperkalemia, hypovolemia and metabolic acidosis. An elevated serum aldosterone level with hyponatremia and hyperkalemia in the absence of elevated 17-OHP level are the key findings to suggest Type 1 PHA. Type 1 PHA was first described in 1958 by Cheek and Perry. ${ }^{4}$ Since then, reports on type 1 PHA have been published and genetic analysis has recently identified two different forms of type 1 primary PHA: Renal PHA 1 or autosomal dominant-PHA and Systemic PHA 1 or autosomal recessive-PHA $1 .^{1,2,5}$

On the other hand, transient PHA with obstructive uropathy was first described by Rodriguez-Soriano et al., in 1983. ${ }^{6}$ Watanabe in his review documented that all patients were younger than 7 months and $80 \%$ of them suffered from both urinary tract malformation and associated UTI similar to our case. ${ }^{7}$ Some cases of transient PHA in infants with UTI who had urinary tract abnormalities were previously documented. ${ }^{8,9}$ Schoen et al., also discussed that the resolution of all hormonal and electrolyte abnormalities was followed by successful treatment of UTI in infants, which was similar to our case. ${ }^{10}$ Development of aldosterone resistance may be predisposed by the development of UTI complicating urinary tract structural anomalies.

Our case presented with afebrile seizure and dehydration due to diarrhoea and urinary tract infection. The most likely cause of her seizure was hyponatraemia. Diarrhoea usually causes hypokalemic metabolic acidosis with varying levels of serum sodium; hyponatremic or hypernatremic or normal depending on the loss of water and sodium. However, the result of her investigations revealing hyponatremic hyperkalemic metabolic acidosis had alerted us that there might be underlying urinary and endocrine pathologies. Thus, we proceeded with further laboratory and imaging studies to obtain a final diagnosis. Normal 17 hydroxyprogesterone level and ACTH stimulation test excluded CAH. The diagnosis of type 1 PHA was strongly suggested by an elevated serum aldosterone.

However, prompt diagnosis of transient PHA may be difficult since the aldosterone assay is normally sent to a reference laboratory and takes several days to obtain the results. Measurement of urinary sodium level prior to treatment may reveal excessive urinary sodium excretion which suggests aldosterone deficiency or resistance. Patient's presentation, laboratory studies, the existence of urinary tract anomalies and family history may help to differentiate between type 1 primary PHA from type 1 secondary, or transient PHA.

Our patient was diagnosed to have urinary tract infection with underlying gross hydronephrosis and hydroureter due to grade 5 vesicoureteric reflux on the right side and exhibited transient renal tubular resistance to aldosterone. After aggressive treatment, she has no subsequent recurrent episode of electrolyte disturbances nor urinary tract infection. We hypothesize that renal inflammation may cause transient tubular resistance to aldosterone independent of structural anomaly. The aldosterone resistance leading to salt wasting and high risk of recurrent urinary tract infection if the structural anomaly is not corrected may lead to failure to thrive in the future. However, our patient did not show any sign of failure to thrive with reasonably good growth. Treatment of transient PHA involves sodium chloride replacement, normalization of potassium level, antibiotic therapy and surgical intervention if indicated.

\section{CONCLUSION}

In conclusion, clinicians should have a high index of suspicion in diagnosing transient PHA in an infant presenting with hyponatremia, hyperkalemia and urinary tract infection with or without associated urinary tract anomalies typically if diagnosis of CAH was not found. Furthermore, in infants presenting with salt wasting, urine cultures should be obtained to investigate for underlying UTI.

\section{Acknowledgments}

The authors thank the parents and the patient for giving consent to use the patient's data.

\section{Ethical Consideration}

Patient consent was obtained before submission of the manuscript.

\section{Statement of Authorship}

All authors certified fulfillment of ICMJE authorship criteria.

\section{Author Disclosure}

The authors declared no conflict of interest. 


\section{Funding Source}

None.

\section{References}

1. Cheong HI. Pseudohypoaldosteronism Type 1. J Genet Med. 2013;10(2):81-7. https://doi.org/10.5734/JGM.2013.10.2.81.

2. Geller DS. Mineralocorticoid resistance. Clin Endocrinol (Oxf). 2005;62(5):513-20. PMID: 15853818. https://doi.org/10.1111/j.13652265.2005.02229.x.

3. Hanukoglu A. Type I pseudohypoaldosteronism includes two clinically and genetically distinct entities with either renal or multiple target organ defects. J Clin Endocrinol Metab. 1991;73(5):936-44. PMID: 1939532. https://doi.org/10.1210/jcem-73-5-936.

4. Cheek DB, Perry JW. A salt wasting syndrome in infancy. Arch Dis Child. 1958; 33(169):252-6. PMID: 13545877. PMCID: PMC2012226.

5. Lee SE, Jung $\mathrm{YH}$, Han $\mathrm{KH}$, et al. A case of pseudohypoaldosteronism type 1 with a mutation in the mineralocorticoid receptor gene. Korean J Pediatr. 2011;54(2):90-3. PMID: 21503203. PMCID: PMC3077507. https://doi.org/10.3345/kjp.2011.54.2.90.
6. Rodríguez-Soriano J, Vallo A, Oliveros R, Castillo G. Transient pseudohypoaldosteronism secondary to obstructive uropathy in infancy. J Pediatr. 1983; 103(3):375-80. PMID: 6350553.

7. Watanabe T. Reversible secondary pseudohypoaldosteronism. Pediatr Nephrol. 2003; 18(5):486. PMID: 12736813. https://doi.org/ 10.1007/s00467-003-1104-6.

8. Melzi ML, Guez S, Sersale G, et al. Acute pyelonephritis as a cause of hyponatremia/hyperkalemia in young infants with urinary tract malformations. Pediatr Infect Dis J. 1995;14(1):56-9. PMID:7715992.

9. Krishnappa V, Ross JH, Kenagy DN, Raina R. Secondary or transient pseudohypoaldosteronism associated with urinary tract anomaly and urinary infection: A case report. Urol Case Rep. 2016;8:61-2. PMID: 27516976. PMCID: PMC4976642. https://doi.org/10.1016/ j.eucr.2016.07.001.

10. Schoen EJ, Bhatia S, Ray GT, Clapp W, To TT. Transient pseudohypoaldosteronism with hyponatremia-hyperkalemia in infant urinary tract infection. J Urol. 2002;167(2 Pt 1): 680-2. PMID: 11792953.

Authors are required to accomplish, sign and submit scanned copies of the JAFES Author Form consisting of: (1) the Authorship Certification that the manuscript has been read and approved by all authors, and that the requirements for authorship have been met by each author, (2) the Author Declaration that the article represents original material that is not being considered for publication or has not been published or accepted for publication elsewhere, (3) the Statement of Copyright Transfer|accepted manuscripts become the permanent property of the JAFES and are licensed with an Attribution-Share Alike-Non-Commercial Creative Commons License. Articles may be shared and adapted for non-commercial purposes as long as they are properly cited], (4) the Statement of Disclosure that there are no financial or other relationships that might lead to a conflict of interest. For Original Articles involving human participants, authors are required to submit a scanned copy of the Ethics Review Approval of their research. For manuscripts reporting data from studies involving animals, authors are required to submit a scanned copy of the Institutional Animal Care and Use Committee approval. For Case Reports or Series, and Images in Endocrinology, consent forms, are required for the publication of information about patients; otherwise, authors declared that all means have been exhausted for securing such consent. Articles and any other material published in the JAFES represent the work of the author(s) and should not be construed to reflect the opinions of the Editors or the Publisher.

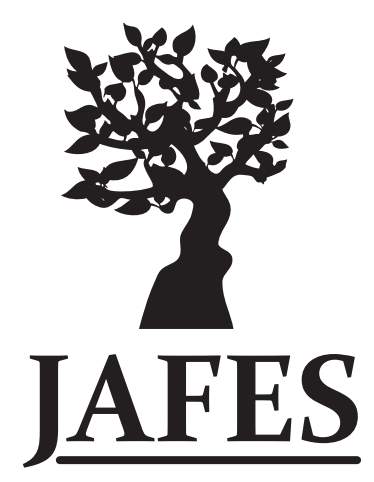

Send your paper to the publication pathway.

Instructions to Authors at www.ASEAN-endocrinejournal.org. 